\title{
Effective visual field size necessary for vertical reading during Japanese text processing
}

\author{
NAOYUKI OSAKA \\ Kyoto University, Kyoto, Japan \\ and \\ KOICHI ODA \\ National Institute of Special Education, Yokosuka, Japan
}

\begin{abstract}
Eye movements were recorded during vertical reading of Japanese text. Saccade length and fixation duration were recorded as a function of moving window size, which varied in length from 1 to 12 characters vertically. Saccade length increased, and fixation duration decreased, as a function of increasing window size. Effective visual field size necessary for vertical reading was found to be approximately 5-6 character spaces in the vertical direction. This span of reading appears to be close to the size during horizontal reading of Japanese text.
\end{abstract}

The direction of writing is different from language to language. The preference of direction depends on the properties of the writing system (Gray, 1956). English as well as other modern European languages are written from left to right, with the order of the lines of print going from top to bottom. Some Semitic languages, such as Hebrew and Arabic, are written from right to left with the order of lines of print going from top to bottom.

There appear to be no clear conclusions that can be drawn about the effect of the direction of print on eye movements or on readers' efficiency in reading (Rayner \& Pollatsek, 1989).

Interestingly enough, modern Japanese can be written and read either vertically or horizontally, although the horizontal order, from left to right, predominates. Historically, Japanese print appeared in columns, with each column being read from top to bottom and the columns read from right to left. But in 1949, the printing policy for public documents was changed, and the left-to-right horizontal direction was recommended. Since then, the left-to-right horizontal direction has come to be more prevalent than the top-to-bottom vertical direction, although the preference for the vertical order is still strong, and a number of documents and books are still printed in columns. ${ }^{1}$ It has been noted that the Japanese writing system offers equal psychological readability for both vertical and horizontal text because the system does not require blank spacing between words (Osaka, 1989).

This research was supported in part by Grant 01510061 from the Ministry of Education, Japan. Thanks are due Hiroshi Ashida for making a part of the computer program. Requests for reprints should be addressed to Naoyuki Osaka, Department of Psychology, Faculty of Letters, Kyoto University, Kyoto 606, Japan.
In Japanese, vertical and horizontal writing are thus functionally equivalent. In the present experiment, we measured the effective visual field for reading vertical Japanese text by using a moving window technique, under the hypothesis that differences in the direction of print would be negligible.

\section{Method}

\section{Apparatus}

In this experiment, we relied on sophisticated eye-movement equipment interfaced with a high-speed computer system connected with the CRT (McConkie \& Rayner, 1975). For eye-movement recording, NAC's eye-mark recorder system (Type V) was employed, coupled with a moving window generator (Osaka \& Oda, 1991). The eye-movement recording system can record the positions of the right/left eye every $33 \mathrm{msec}$, with a sensory array device that is capable of receiving a very narrow infrared $(950 \mathrm{~nm})$ beam reflected from the cornea. Each eye position was recorded in a VTR system via a data output interfacing unit (Type V-99B) (Osaka, 1990).

Current eye-position data in analog format (i.e., $x$-, and $y$-coordinate values) were converted into digital format with a fast $A / D$ unit with 12-bit resolution (Analog Pro A/D unit, Canopus Corp.). Each eye position was fed back, in real time, to construct the rectangular moving window. Current eye position was used to enter the rectangular aperture window in video memory (Hyper-Frame with $768 \mathrm{~K}$ image memory, Digital Arts Corp.), which was superimposed on the text on the analog CRT screen $(640 \times 400$ pixel display, KD-PC854 from NEC Corp.). The text was entered with a word processor and stored on a hard disk (LHD-34HR, Logitec Corp.) in text format. A real-time feedback data processing system was employed with a personal computer (Epson's PC-286VF). When the subject fixated on the text, the subject could see the characters within a certain region around the current fixation point. This technique creates an experimentally defined window region of text for the subject to see on the given fixation. When the subject makes an eye movement, the text in the window area returns to the unreadable masking area and a new window on the text appears at the location of the new fixation. The delay time between the initiation of eye movement and the concomitant window movement was about $26.8 \mathrm{msec}$ on average, measured with a precision timer. That is, the average delay time $=16.5$ (eye-mark recorder) +1.8 (moving window) +8.5 (CRT refresh). 
An optional close-up lens providing a $30^{\circ}$ field was used; it is specially designed for recording eye movements during text reading. A chinrest permitted exact recording.

\section{Materials \\ Eighteen different sets of written Japanese text, each of which con- tained approximately 352 characters, were employed. They consisted of normal easy Japanese text (composed of both kanji and hirakana/katakana characters) selected from the national language texts for high school. The kanji contribution factor (Osaka, 1989) of these texts was 0.26 , and their familiarity level was controlled. The contri- bution factor is the index for evaluating the kanji components in a whole text; it is given by the following formula: $k$ anji contribution factor $=$ (number of kanji characters)/(number of characters in whole text, in- cluding punctuation marks). ${ }^{2}$ Each text set, consisting of 16 columns $\times 22$ characters, subtended a $17^{\circ} \times 20^{\circ}$ visual angle at a viewing dis- tance of $40 \mathrm{~cm}$, and a single character consisted of a $16 \times 16$ pixel matrix and subtended a visual angle of $0.78^{\circ}$. The characters were green ( $x=0.315, y=0.600$, CIE chromaticity values), displayed on a black background.}

\section{Window Size}

Six different window sizes were used: 1, 2, 4, 8, 12 characters, and free viewing. The window length changed along the vertical column but had a fixed width of 2 characters, except in the free window condition, in which no field restriction was used. The position of current fixation, when superimposed on the text, was the center of each window. The separation between character columns was kept at 30 pixels and the vertical intercharacter separation was kept to 1 pixel.

\section{Procedure}

After the calibration period, each subject was asked to read the text from top down silently, as it appeared on the screen. Thirty data points for each window size and subject were measured. Fixations within the area of $1^{\circ}$ of spatial extent and longer than $132 \mathrm{msec}$ ( 4 frames) were selected for the data. Saccade length, including that of the regressive saccade within each vertical column, was collected, but the regressive movement due to return sweep was excluded. Each subject received three sessions for each of the six window conditions, in random order. The first session was the training period; measurements were made during the remaining sessions.

\section{Subjects}

Four college students enrolled in the psychology program were the subjects.

\section{RESULTS AND DISCUSSION}

Mean saccade length, as revealed by character space unit, is shown in Figure 1 as a function of moving window size. A single data point shows a mean of 120 measures across 4 subjects. As window size increased, the mean saccade length increased up to the 12-character window, and it then remained constant for the free field size (22-character window, vertically). Using the method of least squares, a straight line was drawn (saccade length $=0.39 \times$ window size $+1.23, r=.985$ ) between the window sizes of 1 and 12 characters, and the meeting point with the asymptote was estimated. This critical value of about 5.5 character spaces indicates the effective visual field size for vertical reading. Thus, the effective visual field for vertical reading would be about 5-6 characters in size. An analysis of variance (ANOVA) revealed a significant main effect for window size $[F(5,696)=117.74$, $p<.001]$, and there was a significant window $\times$ subject interaction $[F(15,696)=2.43, p<.01]$. However, the main effect of subject did not reach significance

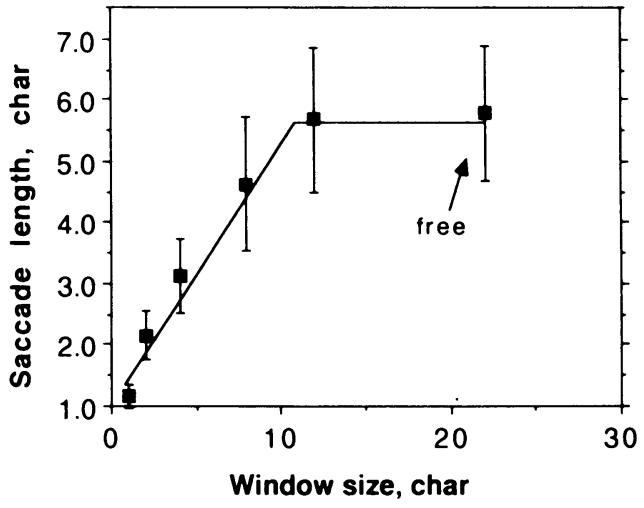

Figure 1. Mean saccade length, in characters, as a function of moving window size; 1-, 2-, 4, 8-, 12-character windows and a 22 character free field were employed. See text for details.

$[F(3,696)=2.00$, n.s. $]$. The means across 4 subjects for the 1-, 2-, 4-, 8-, 12-character and free-window conditions were $1.16,2.15,3.13,4.63,5.68$, and 5.79 characters, respectively. Duncan's multiple range test revealed significant differences among window conditions, excluding the 12-character and free-window conditions $(p<.05)$.

Mean fixation duration as a function of window size is shown in Figure 2. Fixation duration tended to decrease as window size increased. A single data point shows a mean of 120 measures across 4 subjects. An ANOVA revealed significant main effects for window size $[F(5,696)$ $=16.26, p<.001]$ and subject $[F(3,696)=16.32$, $p<.001$ ], as well as a significant window $\times$ subject interaction $[F(15,696)=2.97, p<.001]$. The means across 4 subjects for the 1-, 2-, 4-, 8-, 12-character and free-window conditions were 271, 245, 218, 224, 209, and $208 \mathrm{msec}$, respectively. A Duncan's multiple range test revealed significant differences among window conditions $(p<.05)$, excluding the pairs among 4-, 8-, 12character and free-window conditions.

The effective visual field size ( 5.5 character spaces) estimated here for vertical reading is likely to be quite similar to that estimated on the basis of the average mean sac-

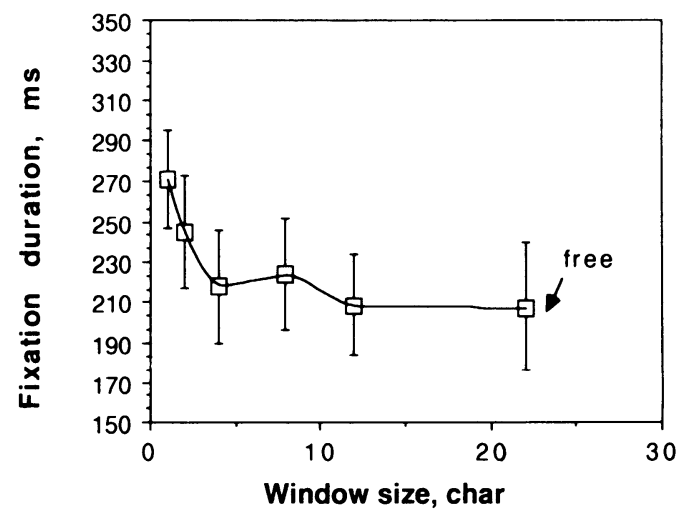

Figure 2. Mean fixation duration as a function of moving window size; 1-, 2-, 4, 8-, or 12-character windows and a 22-character free field. 
cade size (5.8 character spaces; Osaka, 1989) and to that estimated on the basis of the distance from fixation to fixation (3-5 characters; Kambe, 1989). It was found that saccade length was a fixed proportion, 0.39 , of the moving window width. Since the location of the subjects' fixation points bisected the window, the implication is that information ahead of the fixation points was effective. We assumed an asymmetry of the effective visual field-that is, an extension of a few character spaces above and approximately 4-5 character spaces below fixation, as has been reported for horizontal English reading (Rayner, Well, \& Pollatsek, 1980). Motora (1895), using katakana characters, suggested a physiological advantage for horizontal over vertical reading, but the advantage is likely to be too small to be significant (Tanaka, 1916). There appear to be some physiological grounds for believing that horizontal reading may be better; visual resolution falls off more rapidly in the vertical direction than it does in the horizontal direction, and there is a greater ease of eye movement along the horizontal than there is along the vertical. However, in spite of the facts, the evidence that no direction of reading appears to be preferred over any other suggests that these physiological grounds may have a negligible effect on reading (Rayner \& Pollatsek, 1989). Further investigation of the asymmetry of the reading span (McConkie \& Rayner, 1976) and of the reading rate during vertical reading is needed.

\section{REFERENCES}

GraY, W. S. (1956). A study of reading in fourteen languages. In W. S. Gray (Ed.), The teaching of reading and writing: An international study (pp. 53-60). Paris: UNESCO.

KAMBE, N. (1989). The span of the effective visual field during a fixation in reading eye movement. Research Reports of the National
Japanese Language Institute, 10, 59-80. (In Japanese, with English summary)

McConkIE, G. W., \& RAYNER, K. (1975). The span of the effective stimulus during a fixation in reading. Perception \& Psychophysics, 17, 578-586.

McConkie, G. W., \& RAYNer, K. (1976). Asymmetry of the perceptual span in reading. Bulletin of the Psychonomic Society, 8, 365-368.

MotorA, Y. (1895). On the advantage of horizontal and vertical reading. Toyo Gakugei Zassi, 12, 279-286. (In Japanese)

OsAKA, N. (1989). Eye fixation and saccade during kana and kanji text reading: Comparison of English and Japanese text processing. Bulletin of the Psychonomic Society, 27, 548-550.

OsAKA, N. (1990). Spread of visual attention during fixation while reading Japanese text. In R. Groner, G. d'Ydewalle, \& R. Parham (Eds.), From eye to mind: Information acquisition in perception, search, and reading (pp. 167-178). Amsterdam: Elsevier.

OsAKA, N., \& ODA, K. (1991). Moving window for reading and related psychophysical experiments. Manuscript submitted for publication.

Rayner, K., \& Pollatsek, A. (1989). The psychology of reading. Englewood Cliffs, NJ: Prentice-Hall.

Rayner, K., Well, A. D., \& Pollatsek, A. (1980). Asymmetry of the effective visual field in reading. Perception \& Psychophysics, 27, 537-544.

TANAKA, H. (1916). Basic study on language and reading. Tokyo: Meguro. (In Japanese)

\section{NOTES}

1. Vertical reading is adopted in most Japanese school texts-as, for example, in the high school national language textbook.

2. The average kanji contribution factor in the present texts (across 18 texts) is $0.26(S D=0.05)$, and punctuation marks contribute on the average $0.07(S D=0.006)$. Hirakana and Katakana are counted equivalently. Kanji familiarity was controlled by using authorized high school texts. There appears to be no significant difference in readability of the texts within the kanji contribution factor range of $0.2-0.4$, but readability decreases when the factor is less than 0.2 or greater than 0.4 . This indicates that an appropriate mixture ratio of kanji-logograph and kanaphonogram would be crucial for text readability in Japanese.

(Manuscript received January 22, 1991.) 